\title{
In vitro and in vivo assessments of an optimal polyblend composition of polycaprolactone/gelatin nanofibrous scaffolds for Achilles tendon tissue engineering
}

\author{
Sang Jin Lee ${ }^{\mathrm{a}, 1}$, Han-Jun Kim ${ }^{\mathrm{b}, 1}$, Min Heo ${ }^{\mathrm{a}}$, Hye-Rim Lee ${ }^{\mathrm{b}}$, Eun-Ji Choi ${ }^{\mathrm{b}}$, Hyosung Kim ${ }^{\mathrm{b}}$, \\ Donghyun Lee ${ }^{a}$, Rui L. Reis ${ }^{a, c}$, Sun Hee Do ${ }^{b, *}$, Il Keun Kwon ${ }^{a, *}$ \\ a Department of Dental Materials, School of Dentistry, Kyung Hee University, 26 Kyungheedae-ro, Dongdaemun-gu, Seoul 02447, Republic of Korea \\ ${ }^{\mathrm{b}}$ Department of Clinical Pathology, College of Veterinary Medicine, Konkuk University, 120, Neungdong-ro, Gwangjin-gu, Seoul 05029, Republic of Korea \\ c 3B's Research Group - Biomaterials, Biodegradables and Biomimetics, University Minho, Headquarters of the European Institute of Excellence on Tissue \\ Engineering and Regenerative Medicine, Avepark - Parque de Ciência e Tecnologia, Zona Industrial da Gandra, Barco, GMR 4805-017, Portugal
}

\section{A R T I C L E I N F O}

\section{Article history:}

Received 20 December 2018

Received in revised form 11 March 2019

Accepted 20 March 2019

Available online 27 March 2019

\section{Keywords:}

Electrospinning

Polycaprolactone

Gelatin

Highly aligned nanofiber

Tendon tissue engineering applications

\begin{abstract}
A B S T R A C T
In this study, we manufactured various ratios of polycaprolactone (PCL)/gelatin (GE) highly aligned electrospun nanofibrous scaffolds (ENs) to investigate the effects of polymer ratio on tenogenic differentiation activity. For biological assessments, the cell proliferation rate was optimal in the PCL/GE (9:1) group. Interestingly, however, the tenogenic differentiation rate was best for the PCL/GE (7:3) group. From our outcomes, we established that a poly-blending mix of PCL/GE ( $7: 3)$ is a promising ratio for tenogenic differentiation. Thus, our findings may provide for an effective mesh to promote tenogenic differentiation of ENs in future tendon tissue engineering applications.
\end{abstract}

(C) 2019 The Korean Society of Industrial and Engineering Chemistry. Published by Elsevier B.V. All rights reserved.

\section{Introduction}

In the human body, the Achilles tendon is prone to injury due to either strenuous exercise or trauma regardless of intrinsic factors such as age, weight, and gender [1]. Damage to the Achilles tendon is a universal health problem resulting from poor healing of the tendon tissue. For these reasons, many bioengineering researchers have investigated biomaterials as possible therapeutic options for tendon tissue repair [2].

Amongst the developed biomaterials, highly aligned electrospun nanofibrous scaffolds (ENs) have shown promise because they have several advantages. These advantages include matching the anisotropically-aligned geometric structure of tendon extracellular matrix (ECM) and physiologically relevant mechanical properties which may be tuned by controlling the polyblend ratio using both natural and synthetic polymers [3]. For example, a previous report by Yang et al. suggested that an aligned fibrous scaffold made by using polycaprolactone (PCL) and methacrylated gelatin (GE) could work well as a tendon graft scaffold [4]. In

\footnotetext{
* Corresponding authors.

E-mail addresses: shdo@konkuk.ac.kr (S.H. Do), kwoni@khu.ac.kr (I.K. Kwon).

1 These authors equally contributed this work.
}

another study, Wang et al. demonstrated that the aligned polyblend PCL and GE nanofibers could induce tenogenic differentiation of host fibroblasts in a manner superior to that of randomly aligned nanofibers [5]. Poly-blending of synthetic and natural polymers is one of the most promising approaches for providing for aligned scaffolds with desirable properties to be used in tendon tissue regeneration [6]. However, the optimal polyblend ratio of PCL and GE has not yet been fully established. To improve the application of these materials towards tendon tissue engineering, the optimal polyblend balance between PCL and GE should be determined for tendon tissue engineering applications.

In this study, we manufactured various ratios of PCL/GE highly aligned ENs to investigate the effect of ratio on tenogenic differentiation activity. PCL is a semi-crystalline, synthetic, biocompatible, and biodegradable polyester, which is commonly used for ENs in tissue engineering applications and has received approval by the Food and Drug Administration (FDA) [7-9]. It is as an ideal scaffold material for tendon tissue engineering due to its potent mechanical properties [10]. The other material in the polyblend, GE, is a bio-compatible and biodegradable natural polymer. It has a similar chemical composition to natural collagen $[11,12]$. It has also been used for tendon tissue engineering applications $[4,5]$.

The major aim of this study is to provide for an optimal polyblend composition of $\mathrm{PCL} / \mathrm{GE}$ ENs for use in future tendon 
tissue engineering applications (e.g. growth factor and drug delivery) [13]. A detailed schematic illustration of our scaffold generation method is depicted in Fig. 1. The products developed were analyzed by scanning electron microscopy (SEM), X-ray photoelectron spectroscopy (XPS), and Fourier-transform infrared spectroscopy (FT-IR). As in vitro experiments, the products were evaluated using cell proliferation, real-time polymer chain reaction (RT-PCR), and western blot (WB) to ascertain the efficacy of tenogenic differentiation of human dermal fibroblast cells (hDFs) seeded on the scaffold. Finally, an in vivo animal study was performed via ENs implantation in rat Achilles tendon following a simulated crushing injury model. The resultant healing was determined after 8 weeks post-implantation.

\section{Materials and methods}

\section{Materials}

Polycaprolactone (PCL; average MW = $80 \mathrm{kDa}$ ) and Gelatin (GE; porcine skin, type A) were purchased from Sigma-Aldrich (St. Louis, MO). 1,1,1,3,3,3 hexafluoro-2-propanol (HFIP), 1-(3-Dimethylaminopropyl)-3-ethylcarbodiimide hydrochloride (EDC), and $\mathrm{N}$-hydroxysuccinimide (NHS) was purchased from TCI (Tokyo Chemical Industry CO. Ltd., Japan). Ethyl alcohol anhydrous (EtOH, 99.9\%) was purchased from DAEJUNG (DaeJung Chemical \& Metals Co. Ltd., Korea). Human dermal fibroblast (hDF, PCS-201-012) cell line was used purchased from ATCC ${ }^{\circledR}$. Dulbecco's modified Eagle's medium (DMEM), fetal bovine serum (FBS), trypsin-EDTA, antibiotics (penicillin-streptomycin, PS) and Dulbecco's phosphate buffered saline (DPBS) were purchased from Gibco (Rockville, MD, USA). Cell Counting Kit-8 was purchased from Dojindo (Kumamoto, Japan). Qiazol lysis reagent, QuantiTect Reverse Transcription Kit, and Rotor-Gene SYBR Green PCR Kit were purchased from Qiagen (CA, USA). M-PER protein extraction buffer, Halt phosphatase protease inhibitor were purchased from Thermo Fisher Scientific (MA, USA). Protein assay kit and chemiluminescent substrate (Clarity Western ECL substrate) were purchased from Bio-Rad (CA, USA). Polyclonal goat anti-scleraxis antibody (sc87425) and polyclonal rabbit anti-tenascin-C anbitody (SC-20932) were purchased from Santa Cruz Biotechnology (CA, USA). Horseradish peroxidase (HRP)-conjugated secondary antibodies were purchased from Cell Signaling Technology (MA, USA). Neutral buffered formalin (10\% v/v) and hematoxylin and eosin solutions were purchased from BBC Biochemical (WA, USA). Deionizeddistilled water (DDW) was produced by an ultra-pure water system (Puris-Ro800; Bio Lab Tech., Korea). All other reagents and solvents were of analytical grade and used without further purification, unless otherwise specified.

\section{Fabrication of highly aligned PCL/GE ENs via ELSP technique}

The fabrication of highly aligned PCL/GE ENs was performed as previously described with some modification [11,12,14-20]. Briefly, PCL and GE compounds at different blending ratios (10:0, 9:1, 7:3, $5: 5)$ were dissolved in HFIP to produce a $10 \%(w / v)$ solution. For ELSP, the dissolved solution was loaded into a luer-lock syringe attached to a 20 gauge blunt-tip needle. The solutions were electrospun onto an aluminum foil covered rotating windmill mandrel with an applied voltage of $20 \mathrm{kV}$ provided by a highvoltage DC power supply (Nano NC, Korea). The solution feed-rate was $1 \mathrm{~mL} / \mathrm{h}$ by syringe pump (KDS-200, KD Scientific Inc.) and the distance from needle tip-to-collector was maintained at $15 \mathrm{~cm}$. To generate highly-aligned ENs, the mandrel speed was increased to $1800 \mathrm{rpm}$ [16]. After manufacturing, the resultant ENs were dried overnight under vacuum to remove any residual solvent. In order to crosslink the ENs, the scaffolds were immersed in $50 \mathrm{mMEDC}$ and $25 \mathrm{mM}$ NHS in ethanol for $24 \mathrm{~h}$ with gentle shaking followed by washing in ethanol and DDW, sequentially [12]. Finally, the generated scaffolds were lyophilized.

\section{Cell proliferation test of ENs}

Cell proliferation was determined using a Cell Counting Kit-8 (CCK-8) operated according to the manufacturer's instructions. Briefly, four compositions of experimental materials (PCL, PCL/GE (9:1), PCL/GE (7:3), and PCL/GE (5:5)) were placed on the bottom of a 48 -well plate and the hDFs $\left(2 \times 10^{4} /\right.$ well $)$ were seeded onto each. After predetermined time-periods of culture, CCK- 8 reagents

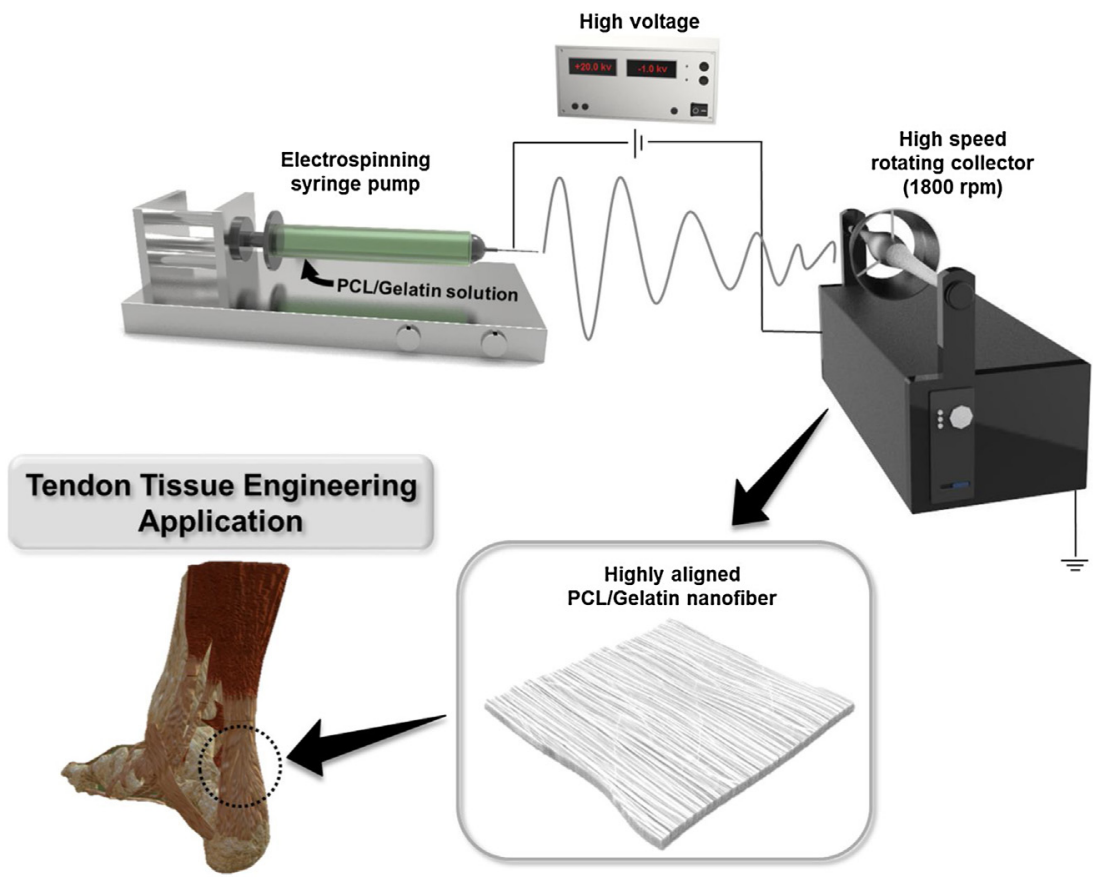

Fig. 1. Schematic illustration of fabrication process of highly aligned PCL/GE ENs via ELSP system. 
were added to the each well and absorbance was measured at $450 \mathrm{~nm}$ using a microplate reader (SUNRISE, TECAN, Salzburg, Austria). F-actin and nuclei were stained as follows. After 7 days of culture, the samples were fixed in 3.7\% formaldehyde solution for $30 \mathrm{~min}$ at $4{ }^{\circ} \mathrm{C}$. After that, the samples were washed several times with PBS and permeabilized with $0.5 \%(\mathrm{v} / \mathrm{v}$ ) Triton X-100 (Sigma Aldrich, USA) in PBS for $10 \mathrm{~min}$. These were subsequently rinsed with PBS three times. Afterwards, the samples were blocked with 1\% BSA for $30 \mathrm{~min}$ and subsequently rinsed with PBS three times. Finally, cell actin filaments were stained by Alexa Fluor ${ }^{\mathrm{TM}} 488$ Phalloidin (Cat. A12379, Invitrogen, USA, dilution 1:200) for $30 \mathrm{~min}$. This was followed by nuclei counterstaining with $4^{\prime}-6-$ diamidino-2-phenylindole (DAPI) for $3 \mathrm{~min}$. The staining procedure was performed at room temperature in the dark. Finally, the dual stained cells on the scaffolds were observed using a confocal laser scanning microscope (CLSM, Eclipse TE2000-E, Nikon, Tokyo, Japan). The obtained images were assayed using Nikon EZ-C1 3.60 software.

\section{Real-time PCR analysis}

The hDFs $\left(2 \times 10^{5}\right)$ were cultured with four different compositions of PCL/GE on $100 \mathrm{~mm}$ culture dishes for 7 and 14 days, respectively. Total RNA was isolated using Qiazol lysis reagent according to the manufacturer's instructions. One microgram of total RNA was transcribed into cDNA with a QuantiTect Reverse Transcription Kit. The primers for the target genes are listed in Table 2. The Rotor-Gene SYBR Green PCR Kit (Qiagen) was used to perform real-time $\mathrm{PCR}$ (initial denaturation, $5 \mathrm{~min}$ at $95^{\circ} \mathrm{C}$; recurring denaturation, $5 \mathrm{~s}$ at $95^{\circ} \mathrm{C}$, and amplification, $10 \mathrm{~s}$ at $60^{\circ} \mathrm{C}$, for 45 cycles). All the mRNA levels were normalized to values of reference genes and the results were calculated as fold changes of the threshold cycle $(\mathrm{Ct})$ value relative to controls using the $2^{-\Delta \Delta C t}$ method. The mRNAs expression of $\beta$-actin was used for the normalization of quantitative expression data.

\section{Western blot analysis}

Total proteins of hDFs ( 7 days and 14 days cultured on four different compositions of PCL/GE) were isolated in M-PER (Thermo Fisher Scientific) extraction buffer with Halt phosphatase protease inhibitor (Thermo Fisher Scientific). The samples were sonicated for $5 \mathrm{~s}$, vortexed, and then centrifuged to recover soluble protein. The protein concentration was determined using a protein assay kit. For each sample, the total protein $(30 \mu \mathrm{g})$ was separated by SDS-PAGE and transferred to PVDF membranes. After blocking with $3 \%$ bovine serum albumin, the membranes were incubated with anti-scleraxis and anti-Tenascin-C antibodies for immunoblot analysis. After washing in TBS/T, the membranes were incubated with horseradish peroxidase (HRP)conjugated secondary antibodies. A CCD camera (ImageQuant LAS 4000, GE Healthcare Life Sciences, PA, USA) was used to detect immunoreactive bands using a chemiluminescent substrate. Quantification was performed with Multi Gauge Version 3.0 software (FUJIFILM, Japan). $\beta$-actin was used for standardizing the protein content per loaded sample.

Table 1

Elemental compositions (\%) of XPS spectra.

\begin{tabular}{llll}
\hline Groups & $\mathrm{C}$ & $\mathrm{N}$ & $\mathrm{O}$ \\
\hline PCL & 77.4 & 0 & 22.6 \\
PCL/GE (9:1) & 74.95 & 2.88 & 22.17 \\
PCL/GE (7:3) & 78.37 & 3.63 & 18.00 \\
PCL/GE (5:5) & 74.22 & 4.26 & 21.52 \\
\hline
\end{tabular}

Table 2

Base sequences of tenogenic primers for real-time PCR.

\begin{tabular}{lll}
\hline & Forward primer sequence & Reverse primer sequence \\
\hline Scleraxis & CGTTGCCCAGGTGCGAGATGTAG & CGAGCGAGACCGCACCAACA \\
Tenascin-C & CAACCATCACTGCCAAGTTCACAA & GGGGGTCGCCAGGTAAGGAG \\
Decorin & TGCTGTTGACAATGGCTCTC & GCCTTTTTGGTGTTGTGTCC \\
$\beta$-actin & TGCCCATCTACGAGGGGTATG & TCCTTAATGTCACGCACGATTTC \\
\hline
\end{tabular}

\section{In vivo implantation in rat model}

Animal experiments were carried out using 10-week-old, male Sprague-Dawley (SD) rats, weighing an average of 300-350 g (Young Bio, Seongnam, Korea). All animal experiments were approved by the Institutional Animal Care and Use Committee of Konkuk University (KU17129). Surgical procedures were performed under general anesthesia induced by $5 \mathrm{mg} / \mathrm{kg}$ of xylazine $\mathrm{HCl}$ (Bayer Korea, Kyungkido, Korea) and maintained with 1.5-2\% isoflurane (Hana Pharm, Gyeonggi-do, Korea) with $100 \% \mathrm{O}_{2}$. After making a longitudinal skin incision parallel to the tendon fiber, the Achilles tendon was dissected from surrounding tissues. The midcentral area of the exposed tendons was crushed for 5 min using a $100 \mathrm{~g}$ vascular clip (Fine Science Tools, Foster City, CA, USA). After the crushing injury, PCL and PCL/GE (7:3) ENs were applied on the lesion. The animals were divided into four groups: exposure only (Sham), injury only, PCL, PCL/GE (7:3) EN-implanted group. All ENs samples were rinsed with PBS and put in UV sterilizing laminar flow hood prior to in-vivo studies. UV sterilization was performed using UV-C germicidal lamp (UV output: $19.8 \mathrm{~W}$, Sankyo Denki, Japan) for about $2 \mathrm{~h}$ of exposure to the sample. For each group, the highly aligned ENs were implanted around the Achilles tendon of the rats. After 8 weeks of implantation, the Achilles tendon of each animal was removed and stained with hematoxylin and eosin (H\&E) to analyze the tendinous structure.

\section{Analysis equipment}

The morphology of the scaffolds was observed using SEM (Hitachi S-4700, Japan) at an acceleration voltage of $15 \mathrm{kV}$. All samples were sputter-coated with platinum for $10 \mathrm{~min}$. The water contact angle was measured by using the water drop method using a video instrument (Phoenix 150, SEO, Korea). XPS was performed using a K-Alpha 89 (Thermo Electron, UK) to confirm the surface chemistry. Fourier transform infrared spectroscopy (FT-IR, PerkinElmer) spectroscopy was recorded in the range from 500 to 4000 $\mathrm{cm}^{-1}$ to characterize the absorption bands of the PCL, GE, and PCL/ GE ENs.

\section{Statistical analysis}

All values are given as mean \pm standard deviation. Statistical analysis was performed using GraphPad Prism 5.0 (GraphPad Software, San Diego, CA). All statistical analyses were performed using two-way ANOVA with Bonferroni's multiple comparison post-hoc tests. Obtained p-values $\left({ }^{*} \mathrm{P}<0.05, \quad{ }^{* *} \mathrm{P}<0.01\right.$, $\left.{ }^{* * *} \mathrm{P}<0.001\right)$ were considered statistically significant.

\section{Results}

Surface morphology characterization of PCL/GE ENs scaffolds (SEM)

In order to verify the surface morphology of manufactured ENs, the materials were characterized using SEM. These images are shown in Fig. 2a. SEM images show that PCL and various ratio of PCL/GE fibers were well fabricated with a relatively uniform size distribution [21]. Additionally, SEM images revealed that 
(a)

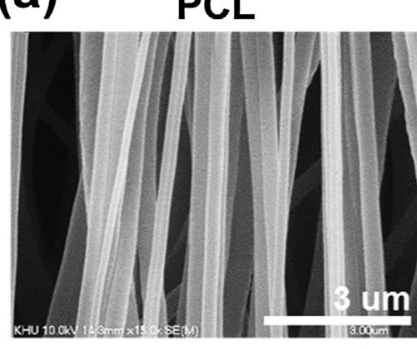

PCL/GE (9:1)

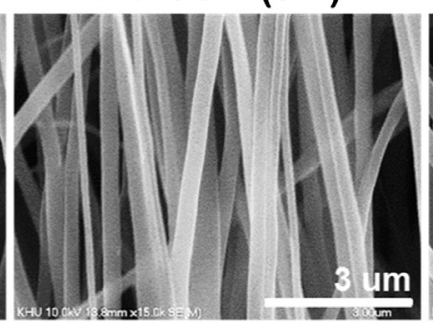

PCL/GE (7:3)

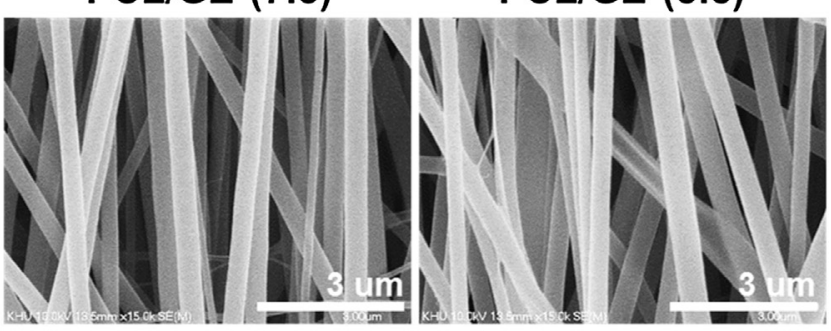

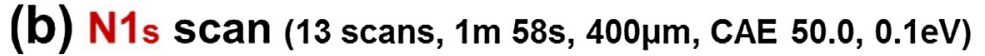

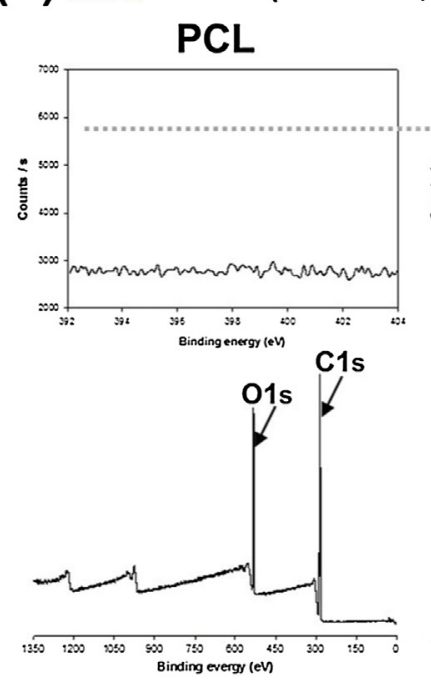

PCL/GE (9:1)
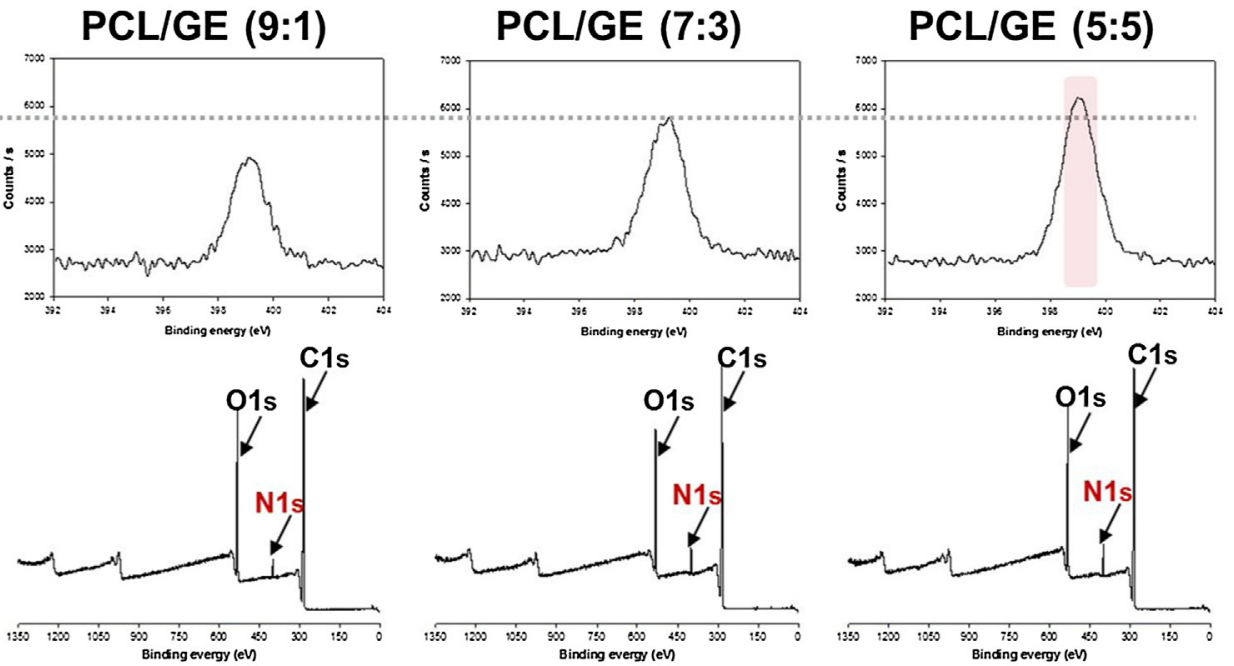

Fig. 2. SEM images (a), and XPS characterization (b) of ENs with different ratios of PCL and GE.

manufactured ENs were bead-less with nanoscale morphology in a highly aligned orientation. Thus, we expected that this highly aligned topography can provide appropriate structural and mechanical support to the geomorphological growth of cells by resembling the native tendon tissue $[5,16]$.

\section{Chemical structures and compositions of ENs scaffolds (XPS and FT-IR)}

To confirm the manufactured ENs chemical structures and compositions, we performed XPS and FT-IR analysis. The results of XPS analysis are shown in Fig. 2b, and Table 1. As can be observed in the XPS result, the spectra of all ENs revealed the presence of carbon and oxygen elements. However, the nitrogen element appeared in PCL/GE groups only and the PCL ENs did not show any nitrogen content. The percent of nitrogen element increased as the content of GE increased. This result is due to the presence of the nitrogen elements in GE. This indicates that GE is successfully incorporated in the PCL/GE ENs [22].

We also verified the chemical structures of the manufactured ENs using FT-IR analysis. As shown in Fig. 3, The asymmetric $\mathrm{CH}_{2}$ stretching $\left(2943 \mathrm{~cm}^{-1}\right)$, symmetric $\mathrm{CH}_{2}$ stretching $\left(2866 \mathrm{~cm}^{-1}\right)$, $\mathrm{C}=\mathrm{O}$ stretching $\left(1728 \mathrm{~cm}^{-1}\right)$, and symmetric $\mathrm{C}-\mathrm{O}-\mathrm{C}$ stretching $\left(1168 \mathrm{~cm}^{-1}\right)$ were found in both PCL and PCL/GE ENs groups. These are characteristic peaks for PCL $[23,24]$. However, the GE characteristic peak at amide I $\left(1640 \mathrm{~cm}^{-1}\right)$ increased with the increase of GE content [22-24]. The FT-IR result coincided with XPS result. Thus, we clearly demonstrated that various ratios of PCL/GE ENs were well fabricated via ELSP system as well as PCL/GE ENs compositions varied depending on the feed ratio of PCL and GE. From these XPS and FT-IR results, we anticipated that different ratios of PCL/GE ENs may affect cell proliferation and differentiation against hDFs.

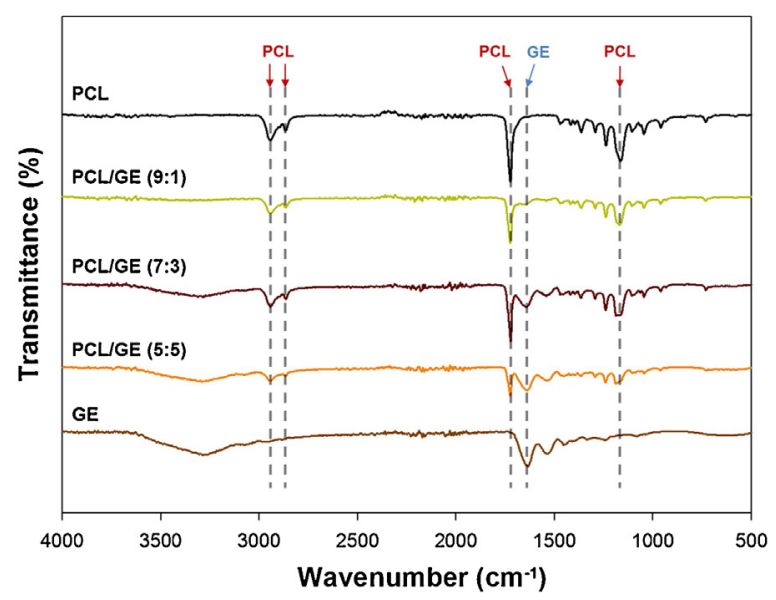

Fig. 3. FT-IR spectra of PCL, GE, and various ratio of PCL/GE ENs. Representative PCL and GE peaks designated as dark red and sky blue arrow, respectively. (For interpretation of the references to color in this figure legend, the reader is referred to the web version of this article).

\section{In vitro cell proliferation response of ENs scaffolds (CCK-8 and IF)}

For measuring the cell proliferation on manufactured scaffolds, we carried out a CCK-8 assay against hDFs for 7 days of culture followed by visualization on ENs at 7 days (Fig. 4). As shown in Fig. 4a, PCL and PCL/GE (7:3) groups showed a similar proliferation rate for 7 days. The PCL/GE (5:5) group showed less proliferation as compared to PCL and PCL/GE (7:3) groups. However, the PCL/GE (9:1) group showed a significantly increased proliferation rate as 


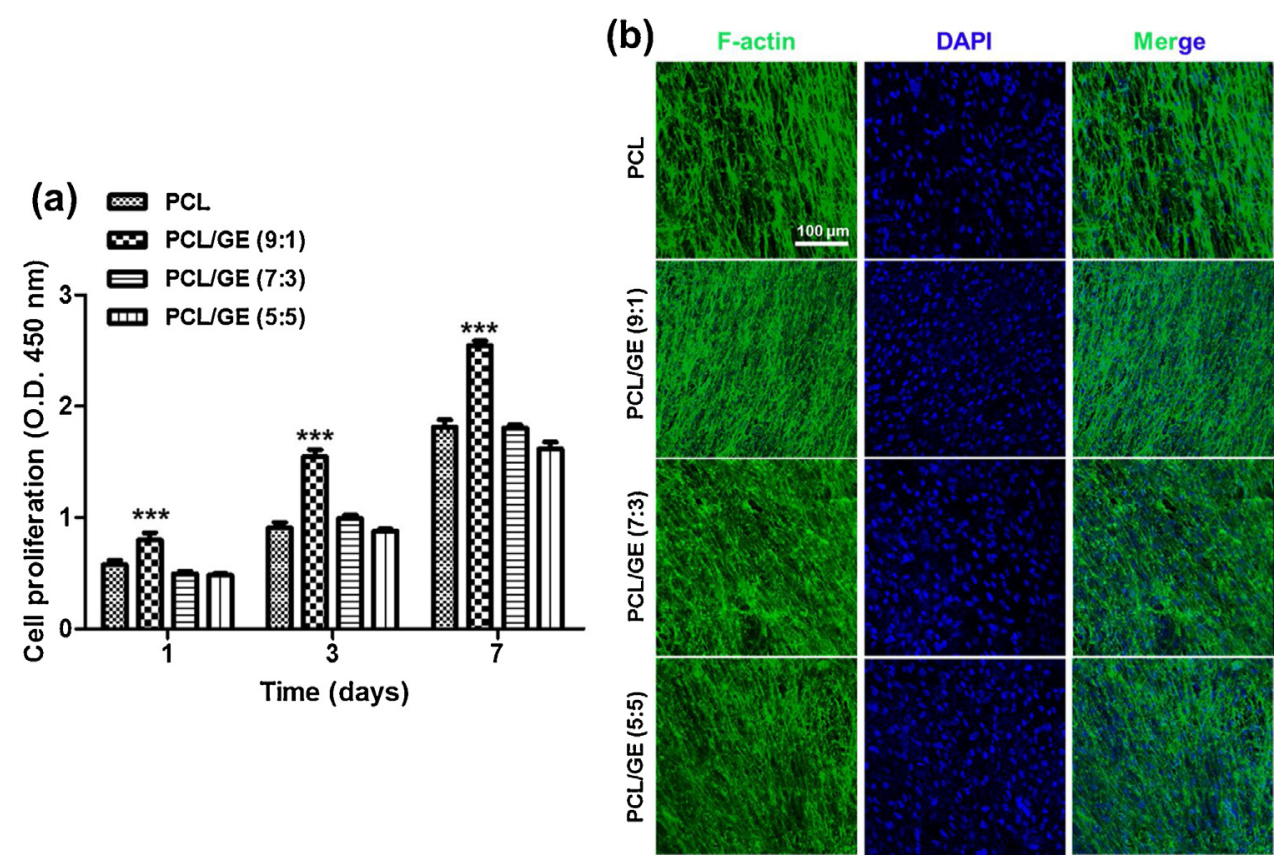

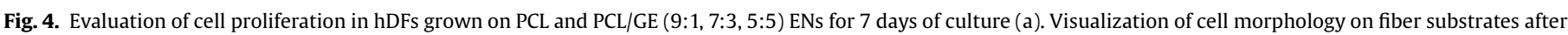

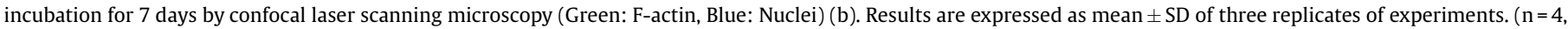
${ }^{* * *} \mathrm{p}<0.001$ compared with other groups). (For interpretation of the references to color in this figure legend, the reader is referred to the web version of this article).

compared to other groups after 7 days of culture. These results correspond with degradation of the GE molecule [25]. After this, the hDFs morphologies were further investigated at 7 days by using CLSM (Fig. 4b). The CLSM micrographs displayed that hDFs grew well on all of the ENs. The hDFs exhibited a parallel alignment morphology on all groups. This morphology indicates that highly aligned ENs provide geometrical guidance for the cells to grow in an elongated environment [26]. This cell morphology directly resembles the microenvironment of native tendon tissue. Furthermore, cell proliferation is best on PCL/GE (9:1) group. Based on this finding, we expected tenogenic differentiation would also lead to different results.

(a) Real-time polymer chain reaction
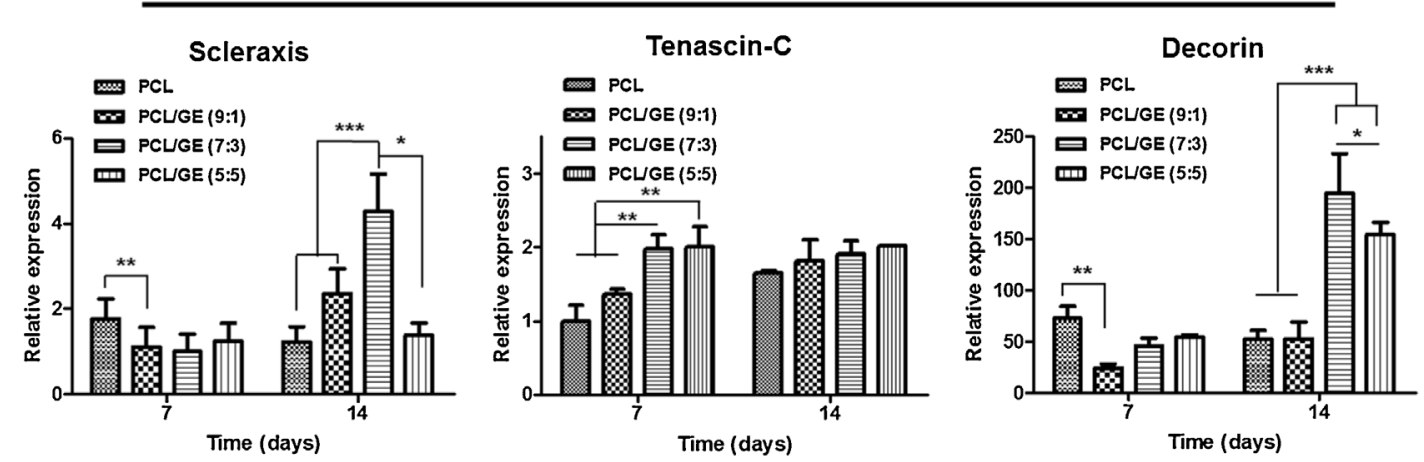

(b) Western Blot analysis
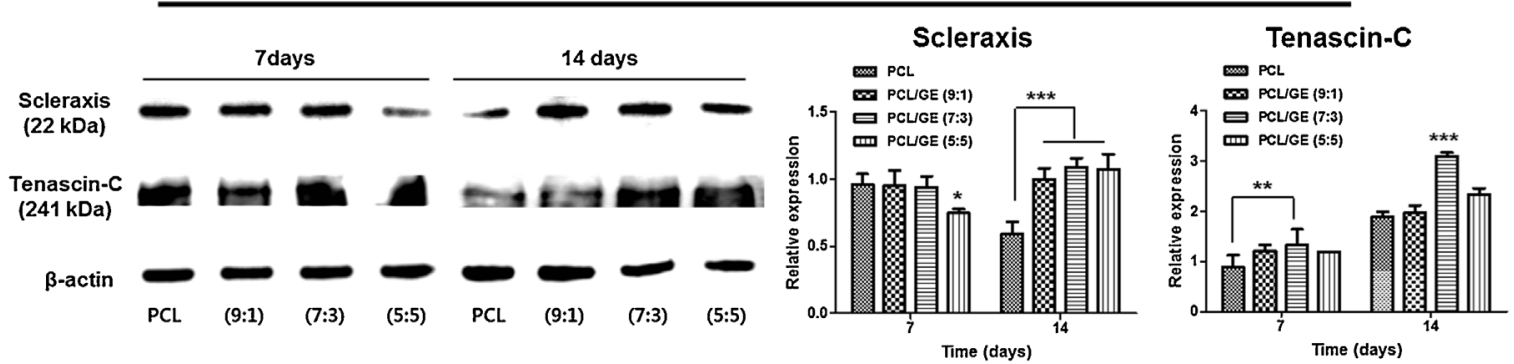

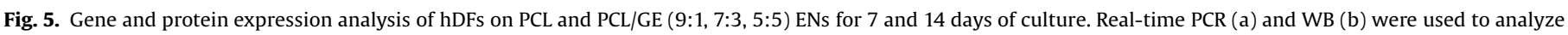
changes in mRNA and protein expression in hDFs. Results are expressed as mean \pm SD of three replicates of experiments. $\left(n=3\right.$, ${ }^{*} \mathrm{P}<0.05$, $\left.{ }^{* *} \mathrm{P}<0.01,{ }^{* * *} \mathrm{P}<0.001\right)$. 
In vitro gene expression and protein analysis of ENs scaffolds (RT-PCR and $W B$ )

To verify the effect of the ENs composition on tenogenesis, quantitative real-time PCR expression analysis was performed at days 7 and 14, as shown in Fig. 5a. The gene expression patterns of experimental groups showed relatively similar patterns for the tenogenic transcription factor, Scleraxis (Scx), and the tenogenic glycoprotein related factor, decorin, over the entire period. The PCL group showed the highest expression levels at day 7 for both Scx and decorin as compared to the other groups. However this scaffold demonstrated the lowest expression level at day 14. On day 14 , Scx expressions were increased with GE composition and peaked in the PCL/GE (7:3) group. As compared to other groups, the PCL/GE (7:3) group showed significantly increased expression levels of decorin. Interestingly, the highest GE composition group, PCL/GE (5:5), showed decreased Scx and decorin expressions as compared to PCL/GE $(7: 3)(\mathrm{p}<0.05)$. On the other hand, the expression of tenogenic glycoprotein related factor, Tenascin- $C$ gene, was increased upon increase in GE content on day 7. However, on day 14, the differences between experimental groups were not significant for this gene.

The protein expression of Scx and Tenascin- $C$ were confirmed by WB analysis. In the PCL group, Scx protein expression was decreased and Tenascin-C expression was slightly increased over time. On the other hand, both $\mathrm{Scx}$ and Tenascin-C protein expression levels were increased in GE containing ENs groups. GE containing ENs groups showed a statistically significant increase in levels of Scx expression as compared to the PCL group at day 14. In the PCL/GE (5:5) group, with the highest GE composition rate, a statistically significant decrease in Scx was observed on Day 7 as compared to the PCL group $(\mathrm{p}<0.05)$. Particularly, Tenascin-C protein expression was higher in the PCL/ GE (7:3) group than in the PCL group throughout the experimental period. These results suggest that the expression of the tenogenic gene/protein varies depending on the GE composition. Higher GE composition is not always favorable for tenogenesis.

In vivo performance of ENs scaffolds in rat model

To determine the tendinous regenerative capacity of the manufactured ENs, PCL and PCL/GE (7:3) ENs were implanted for 8 weeks in a crushed Achilles tendon rat model (Fig. 6). From RT-PCR results, we chose the PCL and PCL/GE (7:3) groups to investigate in the in vivo study. Tendon healing can be classically categorized into three stages including inflammation, fibroplasia, and remodeling phases, with each having some sub-stages. The remodeling phase of tendon healing has three different sub-stages, including alignment stage, maturation stage, and consolidation stage. In particular, the consolidation stage can continue for years [27]. It is well known that mechanical mismatches between the ENs and the Achilles tendon can yield damage to the implantation site, resulting in subsequent acute and chronic inflammation $[28,29]$. PCL group showed that ENs tend to straddle the tendon, due to their hydrophobic characteristics. However, GE-containing PCL/GE (7:3) was easily hydrated and tightly attached to the tendon. However, the degree of inflammation, disorganized regeneration, and dystrophic calcification were different upon the GE composition. In the crushing injury group, the peritendinous inflammatory cell infiltration was still present, and the injured tendinous structure was disorganized and covered by dystrophic calcification. The PCL group also showed localized calcified lesions in the injury site. The PCL/GE (7:3) group showed relatively well aligned regenerated tendon structure without calcification. Taken together, these results suggest that adding GE composition to PCL can alter the physical properties of PCL to increase adherence to the tendon, as well as reduce inflammation and improve tendon-specific regenerative capacity.

\section{Discussion}

There is a need in tendon tissue engineering to establish an optimal environment to promote tenogenic differentiation and healing $[2,30]$. In this case, suitable material selection for manufacturing of a tendon scaffold is necessary for effective treatment of tendon injuries [30]. Additionally, providing for an aligned geometric physical environment is crucial for appropriate cell proliferation and differentiation. This needs to mimic the natural tendon-like cell elongation. To address these issues, we explored PCL/GE composition to induce tenogenic differentiation by using ELSP technique (Fig. 1).

Since the tendons are comprised of mechanically strong and regularly arranged connective tissue bands, there has been a continuing effort to develop treatment technologies based on ELSP (a) Implantation

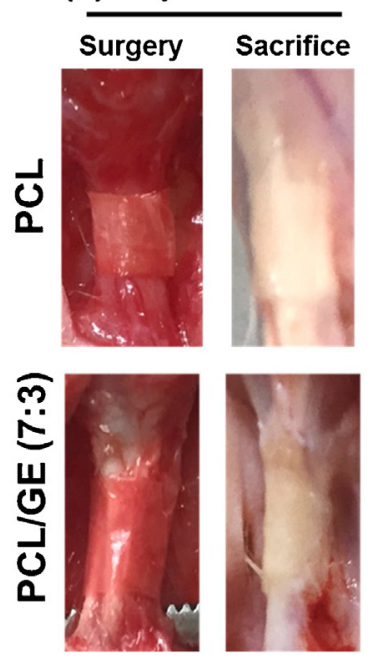

(b) H\&E staining

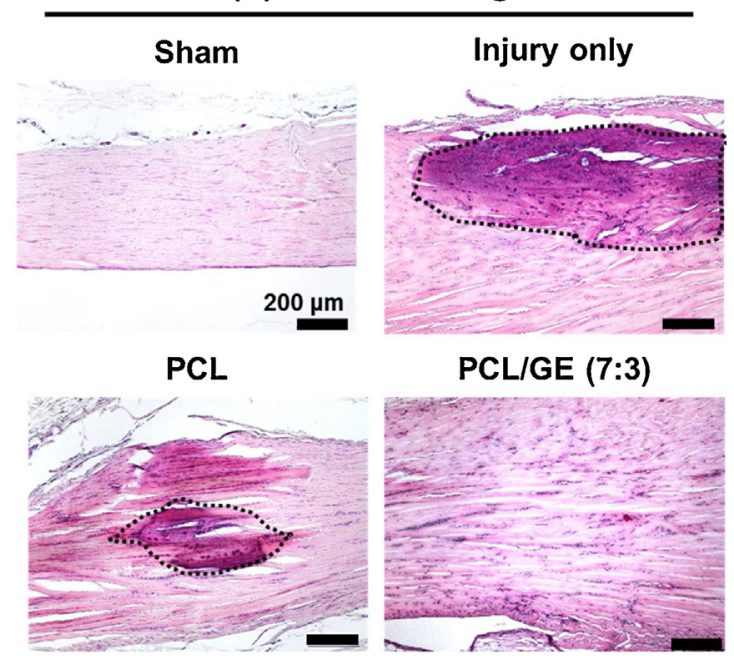

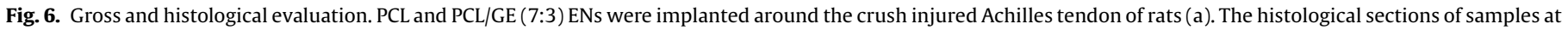

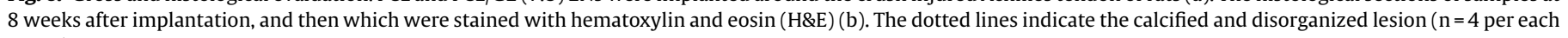
group). 
of tissue engineering scaffolds that can provide for structural and mechanical properties which are similar to the original tendons [3-5]. Despite ELSP's ability to provide for ENs with controlled architectures, there remain a number of challenges for tissue engineering, such as cell adherence, growth, and differentiation $[2,30]$. For decades, research has been actively conducted to determine optimal materials for use in manufacturing ENs [30]. Amongst the materials for tendon regeneration, natural polymers mostly derived from extracellular matrix-based materials, such as collagen and gelatin have come to the forefront [4,31]. Natural polymer has advantages in terms of biodegradation and biocompatibility. It does not provide for the mechanical strength of tendon tissue, however. On the other hand, synthetic polymers, such as PLGA and PCL, show similar mechanical strength and low degradability as tendon tissue. Because of the hydrophobic nature of PCL, PCL EN has several drawbacks including (i) poor wettability, (ii) inadequate cell attachment, and (iii) poor tissue integration. For these reasons, composite scaffolds containing both synthetic and natural polymers have recently been studied. In a previous report, Wang et al. indicated that ENs comprised of a polyblend PCL/GE can promote tenogenic differentiation and that aligned fibers were superior to randomly oriented ENs [5]. Despite this research, an optimal PCL/GE polyblend composition has not yet been fully established. Based on these findings, we produced various ratios of PCL/GE with highly aligned ENs (Fig. 2a). From physico-chemical analysis, we verified that the various ratios of PCL/GE ENs were correctly manufactured (Fig. 2 b and 3 ). We also confirmed that the chemical elements were different depending on the poly-blending ratio (Table 1). From these results, we hypothesized that proliferation and differentiation of cells may have different behavior due to the varying compositions. During in vitro analysis, the different polyblends induced varying cell proliferation and differentiation rates (Figs. 4 and 5). The PCL/GE (9:1) was the best in terms of proliferation of cells as well as PCL/GE (7:3) which was excellent for tenogenic differentiation. This reason is due to the effect of natural gelatin polymer interaction with biological cells [31-33]. In tendon tissue engineering, the combination of a synthetic polymer with a natural polymer can provide for a more effective proliferation and differentiation platform. This improves with increasing interaction between cells and material [34]. Thus, finding the best balance between synthetic and natural materials is a fundamental step towards understanding the intracellular signals that mediate cell proliferation and cell differentiation [2].

Regeneration of tendon tissue is accomplished through a combination of transcription factors, growth factors, and mechanical stimulation. In a normal tendon, the collagen is produced by cells which reside inside the tendon. In this process, Scx acts as a specific biomarker for tendon/ligament cells during the tendon development and regeneration process $[35,36]$. In particular, Scx is known to promote regeneration by increasing the expression of a number of tenogenic differentiation growth factors such as TGF-b2 [37], FGF2 [38], and GDF7 [39]. Extracellular matrix proteins such as decorin (proteoglycan) and Tenascin-C (glycoprotein) also plays a role in organizing the collagen fiber bundle and can be upregulated under mechanical loading during the regeneration phase [40]. In this study, we confirmed that the expression of tenogenic genes and proteins varied depending on the GE composition and that higher GE composition is not always favorable for tenogenesis. In particular, the optimized PCL/GE ENs scaffold induced different expression levels of Scx and decorin. The highest expression was found for the PCL/GE (7:3) composition. Thus, we have established an optimal tenogenic environment.

There has been a great deal of research recently focusing on rapid healing of injured tendons by employing growth factor supplements [41]. In this case, we believe that the optimal fundamental polyblend ratio of tenogenic materials may act synergistically with bioactive molecules. In this respect, our findings will be valuable for further development of effective treatment of injured tendon and ligaments in future tissue engineering applications.

\section{Conclusion}

In this study, we investigated an optimal poly-blending balance of PCL/GE ENs for effective tenogenic differentiation. Using the ELSP technique, the various ratios of highly aligned PCL/GE ENs were manufactured and were characterized by SEM, XPS, and FT-IR analysis. We investigated cellular behavior regarding cell proliferation and tenogenic differentiation on these ENs. Interestingly, cell proliferation was best in the PCL/GE (9:1) group as compared to the other groups. On the other hand, tenogenic differentiation was higher in the PCL/GE (7:3) group as compared with the other groups. During in vivo implantation, the optimized PCL/GE (7:3) group provided for proper physical properties (increased adherence) as well as reduced inflammation and improved regenerative capacity. Thus, our finding may provide for further understanding of the mechanism by which hDFs interact with PCL/GE ENs and are helpful guidance for future tendon tissue regeneration applications. Based on the current research, we plan to immobilize growth/differentiation factor-5 [42] on PCL/GE (7:3) ENs through heparin chemistry to improve tenogenic differentiation [43]. In this case, this preliminary study will pave the way for future investigations.

\section{Author contributions}

The manuscript was written through contributions of all authors. All authors have given approval to the final version of the manuscript.

\section{Notes}

The authors declare no competing financial interest.

\section{Acknowledgement}

This work was supported by the Bio \& Medical Technology Development Program of the National Research Foundation (NRF) \& funded by the Korean government (MSIP\&MOHW) (No. 2017M3A9E4048170).

\section{References}

[1] Z. Yan, H. Yin, M. Nerlich, C.G. Pfeifer, D. Docheva, J. Exp. Orthop. 5 (2018) 1.

[2] J. Lin, W. Zhou, S. Han, V. Bunpetch, K. Zhao, C. Liu, Z. Yin, H. Ouyang, Acta Biomater. 70 (2018) 1.

[3] Y. Wu, Y. Han, Y.S. Wong, J.Y.H. Fuh, J. Tissue Eng. Regen. Med. 12 (2018) 1798.

[4] G. Yang, H. Lin, B.B. Rothrauff, S. Yu, R.S. Tuan, Acta Biomater. 35 (2016) 68.

[5] W. Wang, J. He, B. Feng, Z. Zhang, W. Zhang, G. Zhou, Y. Cao, W. Fu, W. Liu, Nanomedicine 11 (2016) 1055.

[6] M. Mozafari, S. Kargozar, G. de Santiago, M.R. Mohammadi, P. Milan, M. Foroutan Koudehi, B. Aghabarari, M. Nourani, Mater. Technol. 33 (2018) 29.

[7] S.J. Lee, D. Lee, T.R. Yoon, H.K. Kim, H.H. Jo, J.S. Park, J.H. Lee, W.D. Kim, I.K. Kwon, S.A. Park, Acta Biomater. 40 (2016) 182.

[8] S.J. Lee, H.-J. Lee, S.-Y. Kim, J.M. Seok, J.H. Lee, W.D. Kim, I.K. Kwon, S.-Y. Park, S. A. Park, Nanoscale 10 (33) (2018) 15447.

[9] S.J. Lee, M.E. Kim, H. Nah, J.M. Seok, M.H. Jeong, K. Park, I.K. Kwon, J.S. Lee, S.A. Park, J. Colloid. Interface Sci. 537 (2018) 333.

[10] C. Rinoldi, E. Kijeńska, A. Chlanda, E. Choinska, N. Khenoussi, A. Tamayol, A. Khademhosseini, W. Swieszkowski, J. Mater. Chem. B 6 (2018) 3116.

[11] S.J. Lee, D.N. Heo, D. Lee, M. Heo, H. Rim, L.G. Zhang, S.A. Park, S.H. Do, J.-H. Moon, I.K. Kwon, J. Biomed. Nanotechnol. 12 (2016) 2041.

[12] M. Heo, S.J. Lee, D.N. Heo, D. Lee, H.-N. Lim, J.-H. Moon, I.K. Kwon, Appl. Surf. Sci. 432 (2018) 308.

[13] E.J. Carbone, T. Jiang, C. Nelson, N. Henry, K.W.-H. Lo, Nanomedicine 10 (2014) 1691.

[14] S.J. Lee, S.A. Park, D.N. Heo, D. Lee, H.-J. Jang, K.S. Kim, J.-H. Moon, I.K. Kwon, J. Nanosci. Nanotechnol. 16 (2016) 8554.

[15] S.J. Lee, D.N. Heo, J.S. Park, S.K. Kwon, J.H. Lee, J.H. Lee, W.D. Kim, I.K. Kwon, S.A. Park, Phys. Chem. Chem. Phys. 17 (2015) 2996. 
[16] S.J. Lee, M. Heo, D. Lee, D.N. Heo, H.-N. Lim, I.K. Kwon, Appl. Surf. Sci. 424 (2017) 359.

[17] S.J. Lee, D.N. Heo, J.-H. Moon, W.-K. Ko, J.B. Lee, M.S. Bae, S.W. Park, J.E. Kim, D.H. Lee, E.-C. Kim, C.H. Lee, I.K. Kwon, Carbohydr. Polym. 111 (2014) 530.

[18] S.J. Lee, D.N. Heo, J.-H. Moon, H.N. Park, W.-K. Ko, M.S. Bae, J.B. Lee, S.W. Park, E.-C. Kim, C.H. Lee, B.-Y. Jung, I.K. Kwon, J. Nanosci. Nanotechnol. 14 (2014) 7488.

[19] S.J. Lee, D.N. Heo, M. Heo, M.H. Noh, D. Lee, S.A. Park, J.-H. Moon, I.K. Kwon, J. Ind. Eng. Chem. 46 (2017) 273.

[20] D. Lee, S.J. Lee, J.-H. Moon, J.H. Kim, D.N. Heo, J.B. Bang, H.-N. Lim, I.K. Kwon, J. Ind. Eng. Chem. 66 (2018) 196

[21] D. Jaiswal, R. James, N.B. Shelke, M.D. Harmon, J.L. Brown, F. Hussain, S.G. Kumbar, J. Biomed. Nanotechnol. 11 (2015) 2067.

[22] K. Ren, Y. Wang, T. Sun, W. Yue, H. Zhang, Mater. Sci. Eng. C Mater. Biol. Appl. 78 (2017) 324.

[23] X. Yang, J. Yang, L. Wang, B. Ran, Y. Jia, L. Zhang, G. Yang, H. Shao, X. Jiang, ACS Nano 11 (2017) 5737.

[24] V. Pavliňáková, Z. Fohlerová, D. Pavlinák, V. Khunová, L. Vojtová, Mater. Sci. Eng. C Mater. Biol. Appl. 91 (2018) 94.

[25] N. Binulal, A. Natarajan, D. Menon, V. Bhaskaran, U. Mony, S.V. Nair, J. Biomater: Sci. Polym. Ed. 25 (2014) 325

[26] C. Zhang, H. Yuan, H. Liu, X. Chen, P. Lu, T. Zhu, L. Yang, Z. Yin, B.C. Heng, Y. Zhang, Biomaterials 53 (2015) 716.

[27] D.S. Pillai, B.S. Dhinsa, W.S. Khan, Curr. Stem Cell Res. Ther. 12 (2017) 506.

[28] D. Docheva, S.A. Müller, M. Majewski, C.H. Evans, Adv. Drug Deliv. Rev. 84 (2015) 222.
[29] M.T. Rodrigues, R.L. Reis, M.E. Gomes, J. Tissue Eng. Regen. Med. 7 (2013) 673.

[30] G. Walden, X. Liao, S. Donell, M.J. Raxworthy, G.P. Riley, A. Saeed, Tissue Eng. B Rev. 23 (2017) 44.

[31] L. Ghasemi-Mobarakeh, M.P. Prabhakaran, M. Morshed, M.-H. Nasr-Esfahani, S Ramakrishna, Biomaterials 29 (2008) 4532.

[32] Y.-C. Jiang, L. Jiang, A. Huang, X.-F. Wang, Q. Li, L.-S. Turng, Mater. Sci. Eng. C Mater. Biol. Appl. 71 (2017) 901.

[33] R. Zheng, H. Duan, J. Xue, Y. Liu, B. Feng, S. Zhao, Y. Zhu, Y. Liu, A. He, W. Zhang, Biomaterials 35 (2014) 152.

[34] K. Spanoudes, D. Gaspar, A. Pandit, D.I.Zeugolis, Trends Biotechnol. 32 (2014) 474

[35] A.E. Brent, R. Schweitzer, C.J. Tabin, Cell 113 (2003) 235.

[36] R. Schweitzer, J.H. Chyung, L.C. Murtaugh, A.E. Brent, V. Rosen, E.N. Olson, A Lassar, C.J. Tabin, Development 128 (2001) 3855.

37] P. Lui, Y. Rui, M. Ni, K. Chan, J. Tissue Eng. Regen. Med. 5 (2011)e144.

[38] E.D. Ker, A.S. Nain, L.E. Weiss, J. Wang, J. Suhan, C.H. Amon, P.G. Campbell, Biomaterials 32 (2011) 8097.

[39] H. Shen, R.H. Gelberman, M.J. Silva, S.E. Sakiyama-Elbert, S. Thomopoulos, PloS One 8 (2013)e77613.

[40] Y. Liu, C.-W. Suen, J.-f. Zhang, G. Li, J. Orthop. Transl. 9 (2017) 28.

[41] C. Zhang, X. Wang, E. Zhang, L. Yang, H. Yuan, W. Tu, H. Zhang, Z. Yin, W. Shen, X Chen, Acta Biomater. 66 (2018) 141.

[42] T. Wang, B.S. Gardiner, Z. Lin, J. Rubenson, T.B. Kirk, A. Wang, J. Xu, D.W. Smith, D.G. Lloyd, M.H. Zheng, Tissue Eng. Part B Rev. 19 (2012) 133.

[43] S.J. Lee, M.S. Bae, D.W. Lee, D.N. Heo, D. Lee, M. Heo, S.-J. Hong, J. Kim, W.D. Kim, S.A. Park, Carbohydr. Polym. 157 (2017) 1750. 